\title{
Intersections of the Future: Using Fully Autonomous Vehicles
}

\author{
Peter Stone \\ University of Texas at Austin, USA \\ pstone@cs.utexas.edu
}

\begin{abstract}
Artificial intelligence research is ushering in a new era of sophisticated, mass-market transportation technology. While computers can already fly a passenger jet better than a trained human pilot, people are still faced with the dangerous yet tedious task of driving automobiles. Intelligent Transportation Systems (ITS) is the field of study that aims to use artificial intelligence to make transportation safer, cheaper, and more efficient. Recent advances in ITS point to a future in which vehicles themselves handle the vast majority of the driving task. Once autonomous vehicles become popular, autonomous interactions amongst *multiple* vehicles will be possible. Current methods of vehicle coordination, which are all designed to work with human drivers, will be outdated. The bottleneck for roadway efficiency will no longer be the drivers, but rather the mechanism by which those drivers' actions are coordinated. While open-road driving is a well-studied and more-or-lesssolved problem, urban traffic scenarios, especially intersections, are much more challenging.

This talk will address the question: "To what extent and how can a multiagent intersection control mechanism take advantage of the capabilities of autonomous vehicles in order to make automobile travel safer and faster?" First, I will introduce and specify the problem of intersection management as a multiagent system and define a metric by which solutions can be evaluated. Next, I will propose a novel multiagent intersection control mechanism in which autonomous driver agents "call ahead" and reserve space-time in the intersection, pending the approval of an arbiter agent called an intersection manager, which is located at the intersection.
\end{abstract}

